\title{
ESTUDO DO COMPORTAMENTO REOLÓGICO DE FLUIDOS DE PERFURAÇÃO BASE ÁGUA: EFEITO DA CONCENTRAÇÃO DE NaCl
}

\author{
M.M.Vale ${ }^{1}$, F.D.S. Curbelo ${ }^{1}$, G.S.Braga ${ }^{1}$, A.I.C. Garnica ${ }^{1}$
}

${ }^{1}$ Universidade Federal da Paraíba, Centro de Tecnologia, Departamento de Engenharia Química marianamartinsv@hotmail.com

Submetido 06/10/2016 - Aceito 07/02/2017

DOI: $10.15628 /$ holos.2017.5162

\section{RESUMO}

Os fluidos de perfuração são definidos como fluidos de circulação utilizados para auxiliar a operação de perfuração de poços. Especialmente, os fluidos base água apresentam uma série de características ambientalmente mais favoráveis, visto que seus descartes são aceitáveis pelos órgãos ambientais. Porém, a contaminação do fluido com sal originado das formações é um fator crítico para o uso do fluido base água, pois durante o processo de perfuração, o fluido pode sofrer a contaminação salina provocando um aumento repentino na taxa de penetração, na floculação e na viscosidade do fluido. Com isso, este trabalho teve o objetivo estudar a influência da concentração do sal $(\mathrm{NaCl})$ na reologia de fluidos de perfuração base água. Com isso, foram formulados dois fluidos: FP1, com concentrações de $\mathrm{NaCl}$ de 2,61,
$2,87,3,12,3,37,3,62,4,0 \%$ em massa e FP2, com suspensão de bentonita previamente hidratada e $4 \%$ em massa de $\mathrm{NaCl}$. Verificou-se que, o aumento da concentração de $\mathrm{NaCl}$ provocou um aumento em quase todos os parâmetros reológicos do FP1 (viscosidade aparente, forças géis iniciais e finais), causando a sedimentação das partículas sólidas do fluido na concentração de $4,0 \%$ de $\mathrm{NaCl}$, devido à sensibilidade da argila bentonita aos íons de sódio inibindo sua hidratação. Para o FP2, o uso da argila hidratada promoveu uma redução dos parâmetros reológicos, quando comparado ao FP1 com $4 \%$ de $\mathrm{NaCl}$, evitando a sedimentação das partículas sólidas e garantindo, assim, sua estabilidade e seu uso em aplicações petrolíferas.

PALAVRAS-CHAVE: fluidos de perfuração, reologia, Cloreto de sódio ( $\mathrm{NaCl})$.

\section{RHEOLOGICAL BEHAVIOR STUDY OF DRILLING WATER BASED FLUIDS: EFFECT OF CONCENTRATION NaCl}

\begin{abstract}
Drilling fluids are defined as circulation fluids used to aid the drilling of wells. Especially, water-based fluids have a number of more environmentally friendly characteristics, as their discards are acceptable to environmental agencies. However, the contamination of the salt fluid from the formations is a critical factor for the use of the water-based fluid, because during the drilling process the fluid can suffer saline contamination causing a sudden increase in the rate of penetration, flocculation and viscosity of the fluid. This study aimed to develop and study the influence of salt concentration $(\mathrm{NaCl})$ on the rheology of water-based drilling fluids. Thus, two fluids were formulated: FP1, with $\mathrm{NaCl}$ concentrations of $2.61,2.87,3.12,3.37,3.62,4.0$
\end{abstract}

mass\% and FP2, with suspension of bentonite previously hydrated and $4 \%$ by mass of $\mathrm{NaCl}$. It was verified that the increase of the $\mathrm{NaCl}$ concentration caused an increase in almost all rheological parameters of FP1 (apparent viscosity, initial and final gels), causing sedimentation of the solid particles of the fluid in the concentration of $4.0 \% \mathrm{NaCl}$, due to the sensitivity of the bentonite clay to the sodium ions inhibiting its hydration. For FP2, the use of the hydrated clay promoted a reduction of the rheological parameters when compared to FP1 with $4 \%$ of $\mathrm{NaCl}$, avoiding the sedimentation of the solid particles and thus guaranteeing its stability and its use in petroleum applications.

KEYWORDS: drilling fluids, rheology, sodium chloride ( $\mathrm{NaCl})$. 


\section{INTRODUÇÃO}

A perfuração de poços é realizada desde tempos remotos com a finalidade de encontrar água. A busca intensiva por petróleo começou no Século XIV com a industrialização e, consequente aumento da demanda mundial por derivados de petróleo. Inicialmente, os poços eram perfurados por métodos à percussão, em baixa profundidade e com o uso de água ou suspensões formadas com argilas locais como fluidos de perfuração. A literatura registra que o uso de aditivos, como tentativa de controlar as propriedades de um fluido de perfuração, ocorreu pela primeira vez no ano de 1921 (Darley e Gray, 1980).

Os fluidos de perfuração, comumente chamados de lamas, podem ser definidos como fluidos de circulação utilizados para auxiliar a operação de perfuração de poços. Segundo Lummus e Azar (1986), os fluidos de perfuração são indispensáveis à indústria do petróleo, sendo o elemento mais importante na operação de perfuração, sendo, usualmente constituídas de duas fases: uma dispersante (água, óleo ou gás) e outra dispersa, cuja complexidade depende da natureza dos produtos dispersos, requisitos e funções necessárias.

Os fluidos base água apresentam uma série de características ambientalmente mais favoráveis em relação aos outros fluidos (Oliveira et al., 2013), visto que seus descartes são aceitáveis pelos órgãos ambientais, apresentam um fácil tratamento e, também, são mais baratos, fato este que abre caminho para a utilização deste tipo de fluido em várias operações no âmbito petrolífero.

A contaminação do sal é um fator primordial para o impasse do uso do fluido base água, pois durante o processo de perfuração, este fluido pode sofrer a contaminação salina, que consiste na dissolução de vários sais na fase aquosa do fluido de perfuração ao entrar em contato com determinadas formações. As principais fontes de sais são os depósitos de evaporitos e os domos de sal, uma vez que podem ser encontrados sob forma de cristais dispersos nas rochas terrígenas ou dissolvidos nas águas contidas nos poros da rocha. Estes sais podem ser simples ou compostos, monovalentes ou polivalentes. O sal mais comum é a halita, mineral com elevado teor de cloreto de sódio ( $\mathrm{NaCl}$ ) (Lomba, 2007).

Quando se perfura evaporitos ou domos salinos com fluido base água a dissolução instantânea provoca um aumento repentino na taxa de penetração, na floculação e na viscosidade do fluido: estes fenômenos são conhecidos por "wash-out" e "thickening", isto é, lavagem da formação e "engrossamento" do fluido, respectivamente. É recomendável iniciar logo o tratamento do fluido assim que um destes fenômenos é detectado (Lomba, 2007).

No presente trabalho, formulou-se um fluido de perfuração base água comum (FP1), a partir de seus constituintes básicos (adensante, viscosificante, controlador de $\mathrm{pH}$, inibidor, redutor de filtrado) com diferentes concentrações de $\mathrm{NaCl}$ (formulação padrão $(2,61), 2,87,3,12$, $3,37,3,62,4,0 \%$ em massa), realizando o estudo reológico dos mesmos. Investigou-se, assim, a influência da concentração de $\mathrm{NaCl}$ no comportamento reológico dos fluidos de perfuração. $\mathrm{Na}$ segunda etapa, como para a concentração de sal de $4 \%$ em massa o fluido (FP1) tornou-se instável e inoperante, pois após 24 de repouso, houve a sedimentação da fase sólida, provocando 
a perda de suas características reológicas; na tentativa de obter um fluido para suprir essas não conformidades uma nova formulação foi proposta, o fluido (FP2).

\section{REVISÃO BIBLIOGRÁFICA}

\subsection{Fluidos base água}

De acordo com Thomas et al., (2004), o critério principal para definir se um fluido é um fluido base água consiste na natureza da água e dos aditivos químicos empregados na preparação do fluido. A proporção entre os componentes básicos e as interações entre eles provoca sensíveis modificações nas propriedades físicas e químicas do fluido. Consequentemente, a composição é o fator primordial a considerar no controle das suas propriedades.

Os fluidos base água podem ser subdivididos em quatro classes: em não inibidos, em que o fluido não passa por tratamento químico; inibidos, em que há tratamento físico e/ou químico do fluido; fluidos com baixo teor de sólidos e fluidos emulsionados em óleo. Os dois últimos são utilizados em condições especiais, pois os fluidos com baixo teor de sólidos são utilizados com o intuito de aumentar a penetração da broca, reduzindo o custo da operação e fazendo com que a perfuração seja realizada com eficiência. Já os fluidos emulsionados em óleo têm o objetivo de evitar perdas de circulação em zonas que apresentam baixa pressão de poros ou fraturas, reduzindo assim, a densidade do sistema (Thomas, 2001).

\subsection{Reologia dos fluidos}

Segundo Machado (2002), a representação gráfica, ou seja, a curva de fluxo mostra como a tensão cisalhante varia em função da taxa de cisalhamento, e esta define o comportamento reológico dos fluidos viscosos, sendo a equação matemática entre estas duas variáveis, conhecida como equação de fluxo. Os fluidos viscosos podem ser caracterizados, também, através da relação entre a viscosidade e a taxa de cisalhamento, chamada de curva de viscosidade.

O comportamento de fluxo de um fluido é definido pelos parâmetros reológicos, os quais influenciam diretamente no cálculo de perdas de carga na tubulação e velocidade de transporte dos cascalhos. Estes são determinados considerando um modelo matemático particular, o qual influencia diretamente no cálculo das perdas de carga na tubulação e velocidade de carreamento dos cascalhos. Os modelos mais comuns apresentados na literatura são: Modelo de Bingham ou plástico ideal, Modelo de Ostwald de Waale ou fluido de potência, Modelo de Herschel-Bulkley ou fluido de potência com limite de escoamento ou fluido de potência modificado, Modelo de Casson e Modelo de Robertson-Stiff.

Através do estudo reológico, é possível determinar como o fluido escoará sobre variadas condições de temperatura, pressão e taxa de cisalhamento. No campo, as principais propriedades reológicas de interesse, que se encontram vinculadas ao desempenho do fluido 
são: índice de comportamento; índice de consistência; viscosidade aparente; viscosidade plástica; limite de escoamento e força gel (Thomas, 2004).

\subsection{Viscosidade aparente e plástica}

A viscosidade aparente (VA) de um fluido não newtoniano é, por definição, a viscosidade do fluido como se este apresentasse comportamento newtoniano, sendo obtida, através do reograma, pela união de um ponto qualquer da curva à origem. A viscosidade plástica (VP) é definida como a medida da resistência interna do fluido ao escoamento, resultante da interação dos sólidos presentes, ou seja, a viscosidade plástica é uma medida da fricção resultante do choque das partículas entre si (Amorim, 2002) e depende da concentração de sólidos no fluido. Quanto maior o teor de sólidos, maior a fricção entre as partículas e, consequentemente, maior a viscosidade.

\subsection{Limite de escoamento}

O limite de escoamento (LE) é o segundo componente da resistência ao fluxo. Fisicamente, este parâmetro representa o valor mínimo de tensão cisalhante que deve ser aplicado ao fluido para que este inicie o escoamento (Gray et al; 1980). Também é considerado como uma medida das forças eletroquímicas ou de atração presentes no fluido, resultante das cargas positivas e negativas das superfícies das partículas.

\subsection{Forças Géis}

Alguns fluidos de perfuração são tixotrópicos, isto é, adquirem um estado semirígido quando estão em repouso e voltam a adquirir um estado de fluidez quando estão em movimento. A força gel é um parâmetro de natureza reológica que indica o grau de interação elétrica entre as partículas dispersas.

As medidas das forças géis indicam o grau de tixotropia do fluido. A força gel inicial $\left(\mathrm{G}_{0}\right)$ mede a tensão, em lbf/100ft ${ }^{2}$, para colocar o fluido em movimento, após o mesmo ter sido agitado e mantido em repouso por 10 segundos. É uma medida da resistência do fluido ao início do escoamento. A força gel final mede a tensão, em Ibf/100 ft ${ }^{2}$, para colocar o fluido em movimento, após o mesmo ter sido agitado e permanecido 10 minutos em repouso. É uma medida da resistência do fluido para reiniciar o escoamento quando o mesmo passa certo tempo em repouso. A diferença entre estas duas grandezas indica o grau de tixotropia, ou seja, a capacidade do fluido se gelificar com o tempo, impedindo a sedimentação de partículas sólidas.

\subsection{Modelo de Herschel-Bulkley}

O Modelo de Herschel-Bulkley é considerado o mais completo em comparação aos demais modelos, uma vez que a sua equação engloba três parâmetros, tais como: $\tau_{0}$, 
denominado de limite de escoamento real, Kc, que é o índice de consistência que indica o grau de resistência do fluido mediante o escoamento e $\mathrm{n}$, denominado de índice de comportamento e indica fisicamente o afastamento do fluido do modelo Newtoniano. Este modelo foi criado a fim de obter uma relação mais estreita entre o modelo reológico e as propriedades do fluxo de fluidos pseudoplásticos e dilatantes que apresentam um ponto de cedência. A Equação 1 expressa tal modelo:

$$
=\mathrm{K}^{\mathrm{n}}+0
$$

\section{METODOLOGIA}

A metodologia utilizada para o desenvolvimento deste trabalho foi baseada na norma API (1993). A aditivação do fluido ocorre durante sua preparação nos tanques de lama, ou mesmo durante a operação de perfuração, quando é detectada a necessidade de adaptação ou melhoria de suas propriedades. Cada aditivo possui sua função específica, sendo o custo do fluido de perfuração diretamente proporcional à quantidade e tipos de aditivos utilizados (Amorim et al., 2005).

\subsection{Fluidos de perfuração}

Para estudar a influência do sal em fluidos de perfuração base água, foram formulados dois fluidos: FP1, fluido de perfuração base água com concentrações de $\mathrm{NaCl}$ de 2,61, 2,87, 3,12, $3,37,3,62,4,0$ \% em massa e FP2, fluido de perfuração base água com suspensão de bentonita previamente hidratada, com $4 \%$ em massa de $\mathrm{NaCl}$.

\subsubsection{Fluido de Perfuração base água (FP1)}

Para preparação do FP1, adicionou-se água comum no agitador (Hamilton Beach FannModelo 140) e, posteriormente, a adição dos diferentes componentes: goma xantana, HP-amido, Hidróxido de sódio $(\mathrm{NaOH})$, argila ativada, cloreto de sódio $(\mathrm{NaCl})$, triazina e baritina. Os aditivos foram inseridos no agitador, sob agitação máxima, para assim completar a composição do fluido, conforme as etapas abaixo:

1- Adicionou-se água comum ( $V=350 \mathrm{~mL}$ ) ao copo do Hamilton Beach;

2- Adicionou-se lentamente, sob agitação, a goma xantana, aguardando 10 minutos após a adição;

3- Acrescentou-se, vagarosamente, o HP-amido. Em seguida, aguardou-se 10 minutos. Posteriormente, adicionou-se o Hidróxido de sódio $(\mathrm{NaOH})$ e mediu-se o $\mathrm{pH}$ da solução;

4- Acrescentou-se à solução a argila ativada. Depois, esperou-se 10 minutos e, assim, acrescentou-se o cloreto de sódio $(\mathrm{NaCl})$, sob agitação durante 5 minutos;

5- Adicionou-se triazina (bactericida), deixando homogeneizar por 5 minutos;

6- Finalmente, acrescentou-se a baritina, sob agitação, por 10 minutos. 
Após a preparação do fluido de perfuração base água FP1, adicionou-se as concentrações de $\mathrm{NaCl}$ de modo que, as quantidades dos aditivos (goma xantana, $\mathrm{HP}$-amido, $\mathrm{NaOH}$, argila ativada, triazina e baritina) da formulação inicial, permanecessem constantes. $\mathrm{O} \mathrm{NaCl}$ foi adicionado no fluido de perfuração, sob agitação durante 5 minutos, a uma velocidade constante de 17.000 rpm, no agitador Hamilton Beach Fann-modelo 140, em diferentes concentrações (2,61 a 4,00 \% em massa de $\mathrm{NaCl}$ ). Após cada adição de $\mathrm{NaCl}$, o fluido de perfuração permaneceu em repouso durante $24 \mathrm{~h}$ para posterior realização do estudo reológico.

\subsubsection{Preparação dos Fluidos com suspensões bentoniticas hidratadas (FP2).}

A metodologia utilizada para a preparação do FP2 foi a mesma empregada para o FP1, entretanto, as bentonitas utilizadas na preparação do FP2 foram previamente hidratadas em 50 $\mathrm{mL}$ de água comum, agitando durante $20 \mathrm{~min}$, a uma velocidade de $16.000 \mathrm{rpm}$, no agitador Hamilton Beach.

\subsubsection{Avaliação do tempo de hidratação da Bentonita sódica}

Segundo Melo (2008), a bentonita aumenta a viscosidade do meio com o tempo de hidratação. Este fato pode ser agregado a muitos fatores como natureza do cátion interplanar, carga lamelar, natureza dos sítios geradores de carga e interestratificação. Porém, o fator mais relevante apresentado na literatura costuma ser a natureza do cátion interplanar, uma vez que se o íon sódio, que se hidrata muito facilmente, estiver presente em quantidades apreciáveis, a esmectita apresenta alta capacidade de inchamento; enquanto que, se outros íons, como cálcio ou potássio forem predominantes, sua hidratação, pouco intensa, não permite uma grande separação das camadas (Rossi et al., 2002).

A bentonita utilizada neste trabalho foi a BRASGEL (bentonita sódica ativada), o tempo de hidratação da mesma foi de 24 horas, uma vez que a bentonita sódica se hidrata muito facilmente em virtude dos íons sódio. Após a hidratação da bentonita, a adição de $\mathrm{NaCl}$ no FP2 seguiu o mesmo procedimento adotado no fluido de perfuração FP1.

\subsection{Determinação da massa específica do fluido de perfuração}

As massas específicas dos fluidos de perfuração (FP1 e FP2) (em lb/gal) foram determinadas em uma balança de lama Fann, Modelo 140.

\subsection{Determinação dos parâmetros reológicos}

Os ensaios reológicos dos fluidos de perfuração foram realizados em um viscosímetro Fann modelo 35 , à $25^{\circ} \mathrm{C}$. É importante ressaltar que, todo esse sistema trabalha com uma taxa de cisalhamento controlada e suas deflexões lidas em rotações por minuto, isto é, L600, relaciona-se à leitura referente a 600 rpm, L300 à leitura obtida a 300 rpm, e assim por diante. 
3.3.1 Determinação da viscosidade aparente, viscosidade plástica e limite de escoamento.

A partir das leituras das deflexões a 600 e $300 \mathrm{rpm}$, foram determinados a viscosidade aparente (Equação2), viscosidade plástica (Equação3) e limite de escoamento (Equação 4). 0 procedimento mostrado a seguir é baseado no procedimento da API 13B-1 2003 \& Petrobras N2604 (1998):

Viscosidade aparente $(\mathrm{VA})=\mathrm{L}_{600} / 2$

Viscosidade plástica $(V P)=\mathrm{L}_{600}-\mathrm{L}_{300}$

Limite de escoamento $(\mathrm{LE})=\mathrm{L}_{600}-\mathrm{VP}$

\subsubsection{Determinação das forças-géis}

O viscosímetro não fornece apenas os parâmetros de viscosidade plástica, viscosidade aparente e limite de escoamento, mas também os valores de força gel inicial $\left(G_{0}\right)$ e força gel final $\left(G_{f}\right)$, que correspondem à velocidade de formação e rigidez dos géis formados. As medidas das forças-géis se baseou no seguinte procedimento:

1- $\quad$ Liga-se o motor a $600 \mathrm{rpm}$ por 1 (um) minuto. Desliga-se o motor, posicionando assim, a alavanca de velocidade na posição média, para funcionar a $3 \mathrm{rpm}$. Após 10 segundos, liga-se o motor e efetua-se a leitura da máxima deflexão no mostrador. Anota-se esse valor como $\mathrm{G}_{0}$ (gel inicial) em lbf/100ft ${ }^{2}$.

2- $\quad$ Liga-se o motor a $600 \mathrm{rpm}$ por 1 (um) minuto. Desliga-se o motor, colocando-se a alavanca de velocidade na posição média, para funcionar a $3 \mathrm{rpm}$. Ao completar 10 minutos, ligase o motor e efetua-se a leitura da máxima deflexão no mostrador. Conhece-se essa leitura como $\mathrm{G}_{\mathrm{f}}$ (gel final) em lbf/100ft ${ }^{2}$.

\subsection{Determinação dos parâmetros do modelo de Herschel-Bulkley}

O modelo de Herschel-Bulkley (Equação 1) foi o que mais se adequou aos fluidos de perfuração desenvolvidos neste trabalho. Este modelo engloba três parâmetros: $\tau_{0}$, denominado de limite de escoamento real ou tensão de corte inicial; K, denominado de índice de consistência, que indica o grau de resistência do fluido diante do escoamento; e $n$, denominado de índice de comportamento, que indica fisicamente o afastamento do fluido do modelo Newtoniano, ou seja, se o seu valor se aproxima de 1 (um), então, o fluido se aproxima do comportamento Newtoniano.

O valor de $\tau_{0}$ foi determinado por extrapolação do gráfico tensão versus taxa de cisalhamento, em coordenadas cartesianas e o valor de $\mathrm{K}$ e $\mathrm{n}$ foi determinado através do gráfico de $\left(\tau-\tau_{0}\right)$ versus $\gamma$ em coordenadas logarítmicas. 


\section{RESULTADOS E DISCUSSÃO}

Inicialmente, foram determinadas as massas específicas dos fluidos de perfuração formulados, sendo obtidas por meio da balança de lama. O valor foi de, aproximadamente, 9,1 $\mathrm{lb} / \mathrm{gal}$.

$\mathrm{O} \mathrm{pH}$ foi determinado utilizando o pHmetro (TECNOPON mPA 210) resultando em valores de entre 9,0 e 10,0. Posteriormente, foram realizados os ensaios reológicos nos fluidos de perfuração.

\subsection{Fluido de perfuração FP1}

Observando a Tabela 1, verifica-se que o aumento da concentração de $\mathrm{NaCl}$ no fluido FP1 produziu um aumento em quase todos os parâmetros reológicos estudados. Verifica-se que o fluido tornou-se mais viscoso (viscosidade aparente), na medida em que a concentração do sal aumentou. Percebeu-se, também, que quando a concentração do sal aumentou houve um aumento significativo nas forças géis iniciais e finais, resultando, assim, em uma maior resistência inicial para colocar o fluido em escoamento, como também, uma resistência maior do fluido para reiniciar seu escoamento após certo tempo de repouso.

A Tabela 1 mostra os valores dos parâmetros reológicos obtidos dos Fluidos de perfuração FP1.

Tabela 1: Parâmetros reológicos referentes ao fluido de perfuração FP1.

\begin{tabular}{c|c|c|c|c|c}
\hline $\begin{array}{c}\text { Concentração } \\
\text { de } \mathrm{NaCl}(\% \\
\text { em massa) }\end{array}$ & $\begin{array}{c}\text { VP } \\
\text { (cP) }\end{array}$ & $\begin{array}{c}\text { VA } \\
\text { (cP) }\end{array}$ & $\begin{array}{c}\mathrm{LE} \\
\left(\mathrm{lbf} / 100 \mathrm{ft}^{2}\right)\end{array}$ & $\begin{array}{c}\mathrm{G}_{0} \\
\left(\mathrm{lbf} / 10 \mathrm{ft}^{2}\right)\end{array}$ & $\begin{array}{c}\mathrm{G}_{\mathrm{f}} \\
\left(\mathrm{lbf} / 10 \mathrm{ft}{ }^{2}\right)\end{array}$ \\
\hline 2,61 & 9,75 & 18,37 & 17,25 & 7,5 & 10,0 \\
2,87 & 10,0 & 18,5 & 17,50 & 8,0 & 10,0 \\
3,12 & 10,0 & 18,5 & 17,70 & 9,5 & 13,5 \\
3,37 & 9,5 & 20,5 & 22,0 & 11,0 & 14,5 \\
3,62 & 10,0 & 22,75 & 25,5 & 13,0 & 15,0 \\
4,00 & 9,5 & 24,0 & 29,0 & 15,0 & 17,0 \\
\hline
\end{tabular}

\subsubsection{Curvas de tensão e deformação}

As Figuras 1 e 2 mostram os dados experimentais da tensão versus taxa de cisalhamento, para as concentrações de $\mathrm{NaCl}$ estudadas, e o ajuste ao modelo de Herschel-Bukley. 


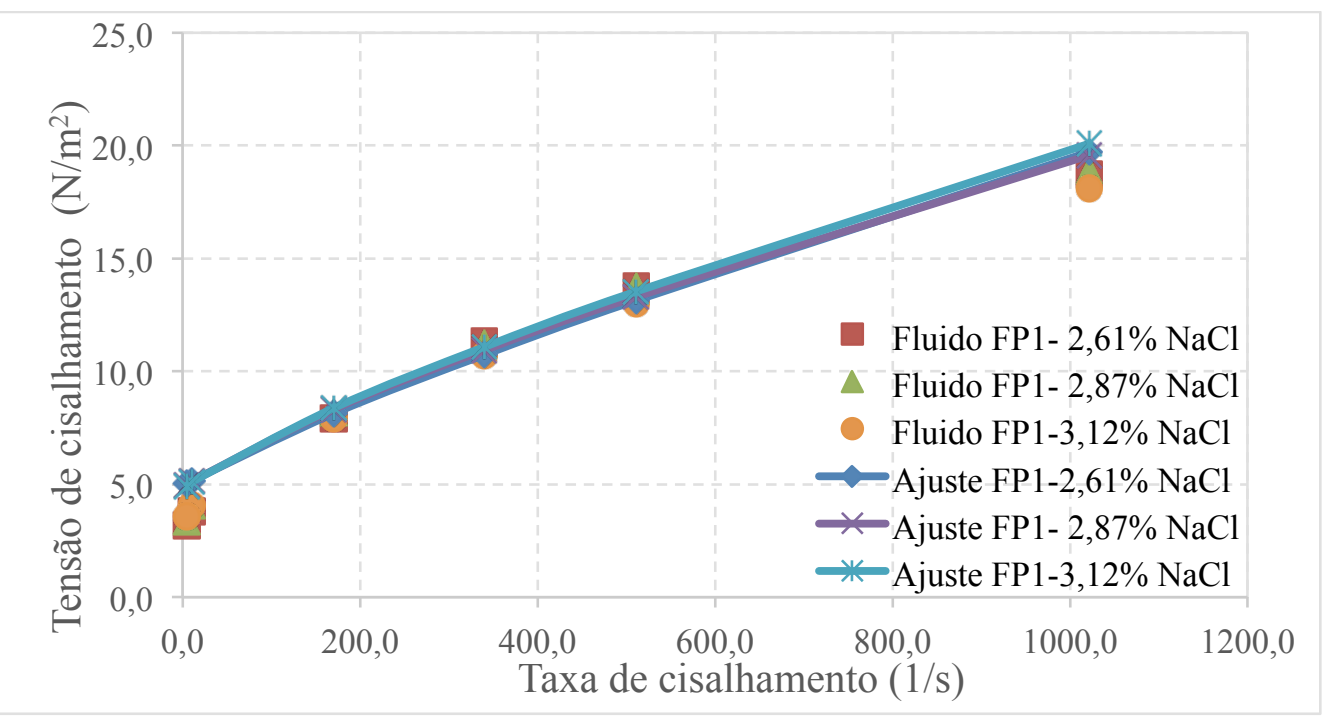

Figura 1: Dados experimentais e ajuste ao modelo Herschel-Bulkley para o fluido FP1, com teor de $\mathrm{NaCl}$ em massa de $2,61 \%$ a $3,12 \%$.

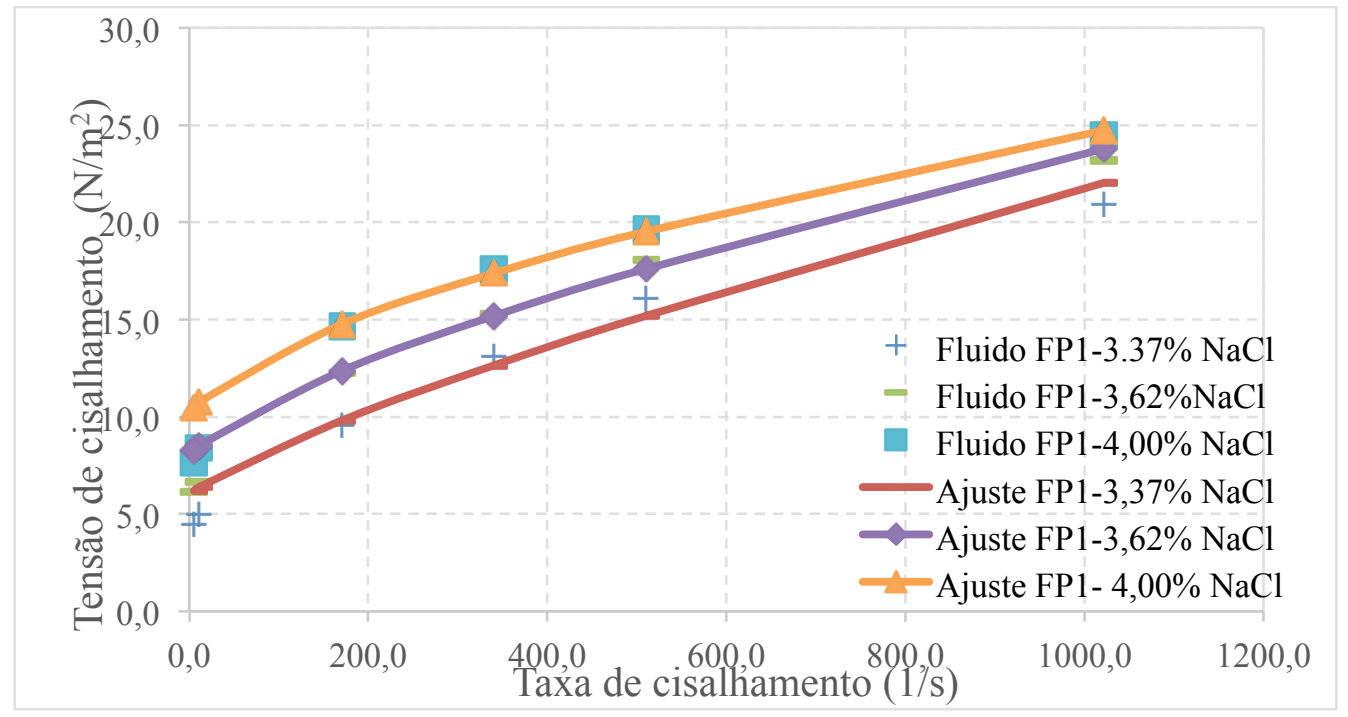

Figura 2: Dados experimentais e ajuste ao modelo Herschel-Bulkley para o fluido FP1, com teor de $\mathrm{NaCl}$ em massa de $3,37 \%$ a $4,00 \%$.

Observa-se nas Figuras 1 e 2 que, os fluidos de perfuração com maiores concentrações de $\mathrm{NaCl}$ apresentaram maiores tensões de corte iniciais (Figura 2), ou seja, o aumento da concentração de $\mathrm{NaCl}$ aumentou o limite de escoamento real. Isto pode ser verificado comparando o comportamento do FP1 com 2,61 \% NaCl (Figura 1), que apresentou tensão de corte inicial igual a 4,9 Pa, com o FP1 com 4,00 \% de $\mathrm{NaCl}$ (Figura 2), que apresentou tensão de corte inicial igual a 9,9 Pa.

Esse resultado é confirmado com os dados das forças géis iniciais e finais e da viscosidade aparente, $15 \mathrm{lbf} / 100 \mathrm{ft}^{2}, 17 \mathrm{lbf} / 100 \mathrm{ft}^{2}$ e $24 \mathrm{cP}$, respectivamente, que são maiores para o FP1 com $4,00 \%$ de $\mathrm{NaCl}$, representados na Tabela 1 . 
A Tabela 2 mostra os dados dos parâmetros do modelo de Herschel-Bulkley.

Tabela 2: Parâmetros do modelo de Herschel-Bulkley.

\begin{tabular}{c|c}
\hline Concentração de $\mathrm{NaCl}$ (\% em massa) & Equações do Modelo de Herschel-Bulkley \\
\hline 2,61 & $=0,044^{0,840}+4,837$ \\
2,87 & $=0,061^{0,793}+4,737$ \\
3,12 & $=0,060^{0,800}+4,713$ \\
3,37 & $=0,064^{0,797}+5,965$ \\
3,62 & $=0,118^{0,708}+7,900$ \\
4,00 & $=0,199^{0,622}+9,897$ \\
\hline
\end{tabular}

Observando as equações apresentadas na Tabela 2, verifica-se que o modelo de HerschelBulkley representou corretamente os dados experimentais dos fluidos estudados, independentemente da concentração do sal utilizada. Verfica-se uma característica pseudoplástica em todas as formulações, uma vez que o índice de comportamento, $n$, encontrase entre $0<\mathrm{n}<1$. Verifica-se também que, o índice de consistência, $K$, aumentou com o aumento da concentração do sal, indicando assim, uma maior resistência ao escoamento do fluido, o que também resultou no aumento da viscosidade aparente e das forças géis iniciais e finais, mostrados na Tabela 1.

A Figura 3 e a Tabela 3 apresentam os resultados da comparação da tensão experimental $(\tau)$ com a tensão calculada pelo modelo de Herschel-Bulkley ( $\tau$ teórico).

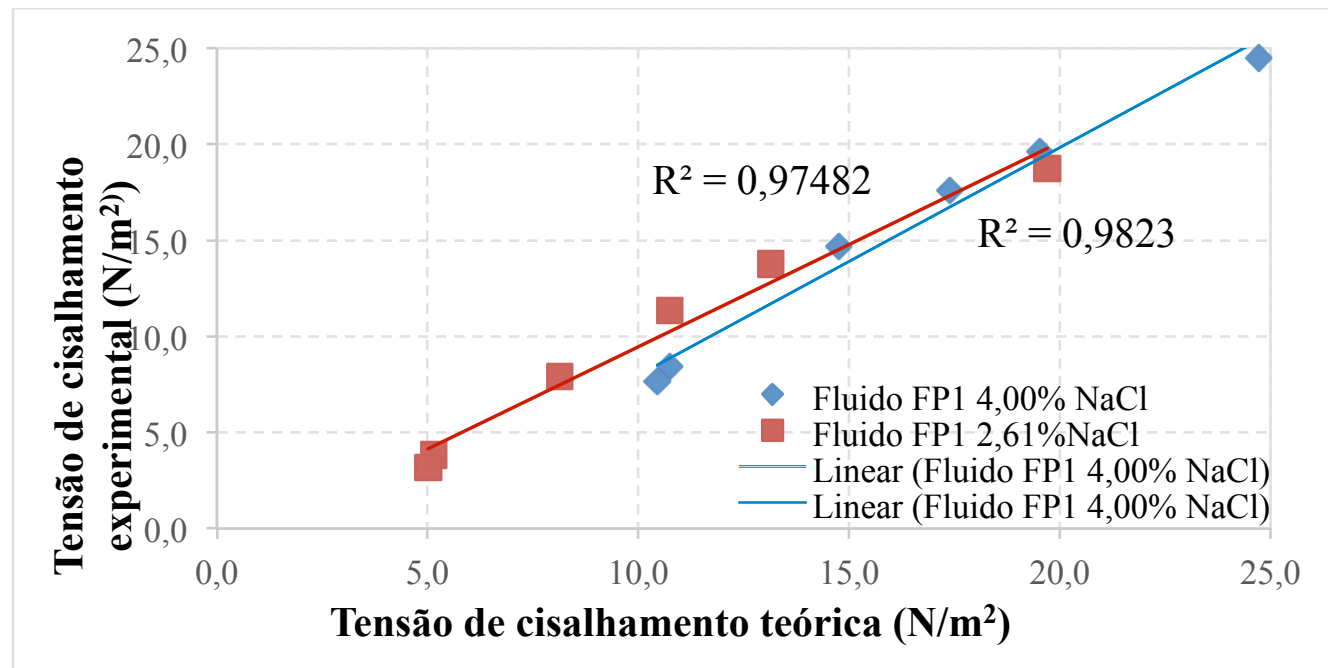


Figura 3: $\tau$ experimental versus $\tau$ teórico do fluido de perfuração FP1 com 2,61\% e 4,00\% de $\mathrm{NaCl}$ em massa.

Tabela 3: Equações das retas geradas nos gráficos de tensão experimental versus tensão teórica.

\begin{tabular}{c|c|c|c|c|c}
\hline \multicolumn{6}{c}{ Concentração de $\mathrm{NaCl}$ (\% em massa) } \\
\hline 2,61 & 2,87 & 3,12 & 3,37 & 3,62 & 4,00 \\
\hline $\mathrm{y}=1,0642 \mathrm{x}-$ & $\mathrm{y}=1,0632 \mathrm{x}-$ & $\mathrm{y}=0,9606 \mathrm{x}-$ & $\mathrm{y}=1,0586 \mathrm{x}-$ & $\mathrm{y}=1,1168 \mathrm{x}-$ & $\mathrm{y}=1,1847 \mathrm{x}$ \\
1,179 & 1,1239 & 0,5425 & 1,2307 & 2,3558 & $-3,8646$ \\
& & & & & \\
$\mathrm{R}^{2}=0,9748$ & $\mathrm{R}^{2}=0,9850$ & $\mathrm{R}^{2}=0,9891$ & $\mathrm{R}^{2}=0,9761$ & $\mathrm{R}^{2}=0,9841$ & $\mathrm{R}^{2}=0,9823$ \\
\hline
\end{tabular}

Verifica-se, através da Figura 3 e da Tabela 3, que o modelo de Herschel-Bulkley reproduziu corretamente os dados experimentais obtidos. Isto foi comprovado através da comparação do valor da tensão de cisalhamento experimental $(\tau)$ com o valor obtido pelo modelo de Herschell-Bulkley, ou seja, $\tau$ teórico.

A Figura 4 representa o comportamento da viscosidade coma a taxa de cisalhamento:

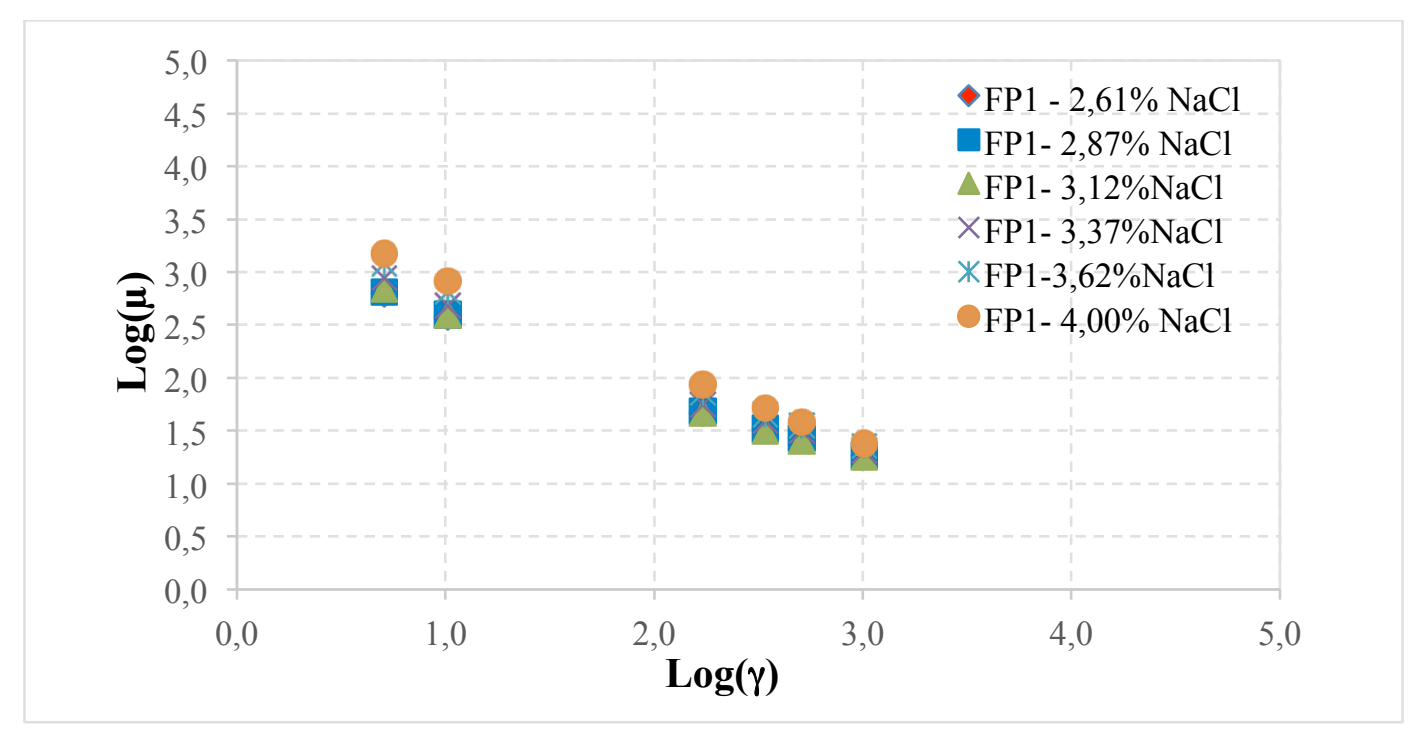

Figura 4: $\log \mu$ (viscosidade) $\times \log \gamma$ (taxa de cisalhamento) para o fluido FP1.

Observando a Figura 4, verifica-se um decréscimo acentuado da viscosidade aparente quando a taxa de cisalhamento é aumentada para todas as concentrações de sal estudadas, característica esta de fluidos pseudoplásticos. Este comportamento é importante para o fluido de perfuração, visto que com o aumento da velocidade de escoamento do fluido dentro da coluna de perfuração, tem-se um aumento da taxa de cisalhamento e, consequentemente, uma diminuição da viscosidade deste fluido, pois dentro da coluna necessita-se de certa facilidade para o fluido escoar.

Apesar do fluido de perfuração FP1 apresentar um comportamento pseudoplástico, verificou-se, de forma geral, que o aumento da concentração do sal pode comprometer o uso deste fluido, devido ao aumento das forças géis iniciais e finais e da viscosidade aparente. Especificamente na formulação com 4,00 \% de sal, houve a sedimentação das partículas sólidas do fluido, após $24 \mathrm{~h}$ de repouso, como mostra a Figura 5, tornando este instável e inoperante. 


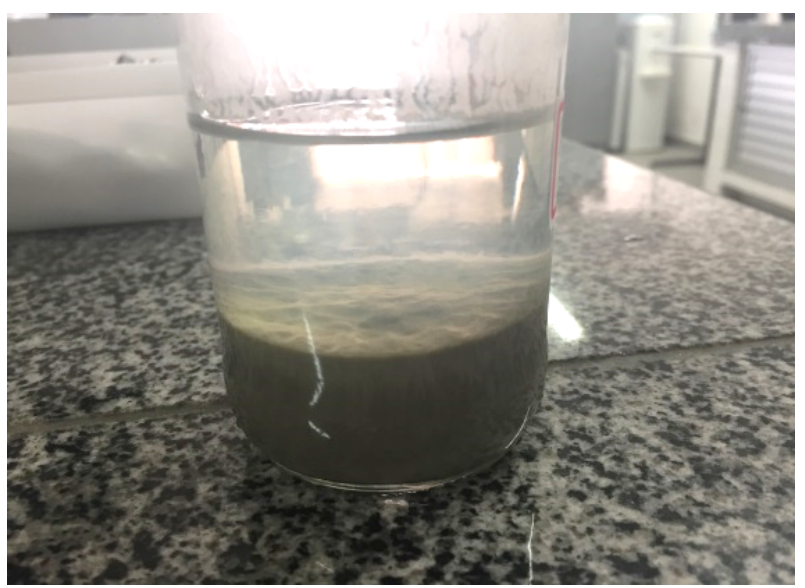

Figura 5: Fluido de perfuração FP1 com 4,00\% de NaCl após 24 h de formulação.

Conforme Amorim et al., 2005, esta perda da estabilidade e, consequentemente, das suas propriedades, deve-se à sensibilidade do sistema argila bentonita-água, aos íons de $\mathrm{Na}^{+}$que, quando presentes, inibem a hidratação das partículas de argila, aumentando a força atrativa entre elas, favorecendo interações do tipo face-a-face, ou seja, promovendo a floculação do sistema e, consequentemente, a perda das propriedades reológicas.

Tendo em vista o exposto anteriormente, foi proposto uma nova formulação do fluido de perfuração, denominada de FP2, mantendo os mesmos constituintes básicos nas mesmas proporções do $\mathrm{FP1}$, com percentual de 4,00 \% em massa de $\mathrm{NaCl}$, porém nesta formulação, a argila (bentonita) foi hidratada previamente à formulação do fluido.

\subsection{Análise comparativa dos fluidos FP1 e FP2}

A Tabela 4 mostra os valores dos parâmetros reológicos para os fluidos de perfuração FP1 e FP2 com 4,00\% em massa de $\mathrm{NaCl}$.

Tabela 4: Análise comparativa dos parâmetros reológicos dos Fluidos de Perfuração FP1 e FP2 com 4,00\% NaCl.

\begin{tabular}{c|c|c|c|c|c|c}
\hline Fluidos & $\begin{array}{c}\text { VP } \\
(\mathrm{cP})\end{array}$ & $\begin{array}{c}\mathrm{VA} \\
(\mathrm{cP})\end{array}$ & $\begin{array}{c}\mathrm{LE} \\
\left(\mathrm{lbf} / 100 \mathrm{ft}^{2}\right)\end{array}$ & $\begin{array}{c}\mathrm{G}_{0} \\
\left(\mathrm{lbf} / 100 \mathrm{ft}^{2}\right)\end{array}$ & $\begin{array}{c}\mathrm{G}_{\mathrm{f}} \\
\left(\mathrm{lbf} / 100 \mathrm{ft}^{2}\right)\end{array}$ & $\tau_{0}$ \\
\hline FP1 & 9,5 & 24,0 & 29,0 & 15,0 & 17,0 & 9,9 \\
FP2 & 11,0 & 19,0 & 16,0 & 9,0 & 11,0 & 5,5 \\
\hline
\end{tabular}

Observando os parâmetros reológicos da Tabela 4, percebe-se que hidratação da argila bentonita resultou em uma redução significativa dos parâmetros reológicos, como indicados pelos valores da força gel inicial e final e a viscosidade aparente, propiciando, assim, melhores condições para o uso deste em perfuração de poços de petróleo.

A Figura 6 representa os dados experimentais da tensão versus taxa de cisalhamento e o ajuste feito pelo Modelo de Herschel-Bulkley para os dois tipos de fluidos: FP1 e FP2 com 4,00\% em massa de $\mathrm{NaCl}$. 


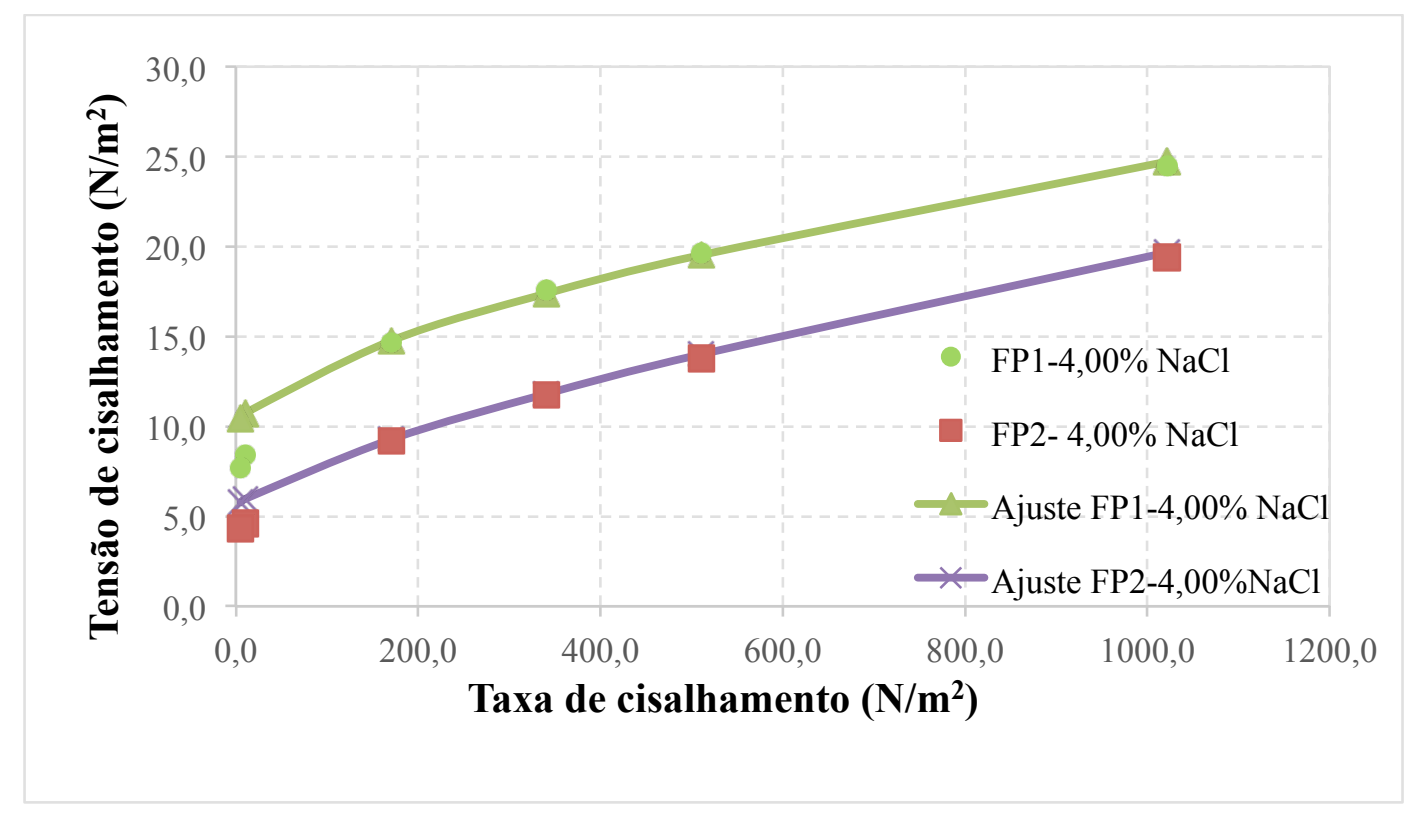

Figura 6: Dados experimentais e ajuste do modelo Herschel-Bulkley para os fluidos de perfuração FP1 e FP2 com $4,00 \%$ em massa de $\mathrm{NaCl}$.

Através da análise da Figura 6, observa-se que o fluido FP2 apresentou um limite de escoamento real menor que a do fluido FP1, com valores de 5,5 e 9,9 lbf/100ft, respectivamente. Estes valores indicam como o fluido FP2 apresentou uma menor resistência inicial para escoar, como também, uma menor resistência para reiniciar seu escoamento após certo tempo de repouso. Este resultado também é confirmado pela diminuição da viscosidade aparente e das forças géis iniciais e finais, apresentadas na Tabela 4.

A Tabela 5 mostra as equações obtidas através dos parâmetros do modelo de HerschelBulkley para os dois fluidos, FP1 e FP2, com concentração de 4,00 \% NaCl em massa.

Tabela 5: Equações do modelo de Herschel-Bulkley para os fluidos FP1 e FP2 com 4,00 \% NaCl.

\begin{tabular}{cr}
\hline & Equações do modelo de Herschel-Bulkley \\
\hline FP1 & $=0,199^{0,6221}+9,897$ \\
FP2 & $=0,083^{0,737}+5,490$ \\
\hline
\end{tabular}

Ao verificar os valores das equações apresentadas na Tabela 5, observa-se que o fluido FP2 apresentou também, características pseudoplásticas, uma vez que, o índice de comportamento foi de $n=0,737$ e encontra-se entre $0<n<1$. Observando o índice de consistência, K, percebeu-se que seu valor para o fluido FP2 foi menor (0,083 Pa.s) do que para o fluido FP1 (0,1991 Pa.s), o que significa que o fluido FP2 apresentará menor resistência ao escoamento, já confirmado com a análise da Figura 6, e que ,também, pode ser comprovado por 
uma menor viscosidade aparente $(19 \mathrm{cP})$ e menores valores das forças géis iniciais $\left(9,0 \mathrm{lbf} / 100 \mathrm{ft}^{2}\right)$ e finais (11 lbf/100ft ${ }^{2}$ ) (Tabela 4).

Pode-se afirmar que, a nova formulação do fluido (FP2), com a hidratação prévia da argila bentonita, melhorou suas propriedades reológicas e evitou a sedimentação das partículas sólidas, garantindo, assim, sua estabilidade e seu uso.

\section{CONCLUSÕES}

1- O aumento da concentração de $\mathrm{NaCl}$ no Fluido $\mathrm{FP} 1$, promoveu, um aumento em quase todos os parâmetros reológicos estudados. Acarretando numa resistência inicial maior para colocar o fluido em escoamento, assim como, uma resistência maior do fluido para reiniciar seu escoamento após certo tempo de repouso.

2- O modelo de Herschel-Bulkley representou corretamente os dados experimentais dos fluidos estudados, independentemente da concentração do sal, observando-se uma característica pseudoplástica em todas as formulações, em que, o índice de consistência, $K$, aumentou com o aumento da concentração do sal.

3- Para a concentração de sal de 4,0 \% em massa no FP1, o fluido tornou-se inoperante, pois após 24 horas de repouso, houve a sedimentação da fase sólida, provocando a perda de suas características reológicas.

4- A nova formulação do fluido, fluido FP2, com a hidratação da bentonita, melhorou suas propriedades reológicas e evitou a sedimentação das partículas sólidas, garantindo, assim, sua estabilidade e seu uso em aplicações petrolíferas.

\section{REFERÊNCIAS}

Amorim, L. V., Farias, K. V., Viana, J. D., Barbosa, M. I. R., Pereira, E., França, K. B., ... \& Ferreira, H. C. (2005). Fluidos de perfuração à base de água. Parte I: Efeitos de aditivações poliméricas nas propriedades reológicas. Cerâmica, 51(318), 128-138.

Amorim, L. V., Gomes, C. M., Silva, F. L. H., \& Ferreira, H. C. (2002). Comportamento reológico de dispersões de argilas bentoníticas: efeitos do tipo de ferramenta, velocidade e tempo de agitação. Cerâmica, 48(308), 75-85.

API, Norma API Recommended Practice 13B-1 (2003).

de Melo, K. C. (2008). Avaliação e modelagem reológica de fluidos de perfuração base água (Doctoral dissertation, Universidade Federal do Rio Grande do Norte).

GRAY, George Robert; DARLEY, Henry CH; ROGERS, Walter Francis. Composition and properties of oil well drilling fluids. Gulf Publishing Company, Book Division, 1980.

Guimarães, I. B., \& ROSSI, L. D. S. (2007). Estudo dos constituintes dos fluidos de perfuração: proposta de uma formulação otimizada e ambientalmente correta. 4o PDPETRO. 
Harvey, F. (1990). Fluid program built around hole cleaning, protecting formation. Oil and Gas Journal;(USA), 88(45).

Lomba,R. Engenharia de Petróleo. Apostila do curso de Engenharia de Petróleo. Pontifícia Universidade Católica de São Paulo, Brasil, 2007.

Lummus, J. L., \& Azar, J. J. (1986). Drilling fluids optimization: a practical field approach.

Machado, J. C. V. (2002). Reologia e escoamento dos fluidos: ênfase na indústria do petróleo. Interciência.

OLIVEIRA, A. K. C., ABRAÃO, P. C., \& ABRAÃO, R. C. (2013, June). Revisão sobre a formulação de um fluido de perfuração à base água. In IX Congresso de Iniciação Científica do IFRN.

Petrobras, Viscosificantes para Fluido de Perfuração Base de Água na Exploração e Produção de Petróleo, Especificação, N-2604 (1998).

Thomas, J. E. (2001). Fundamentos de engenharia de petróleo. Interciência.

Thomas, J.E. Fundamentos de Engenharia de petróleo, 2aedição, Editora Interciência, 2004. 